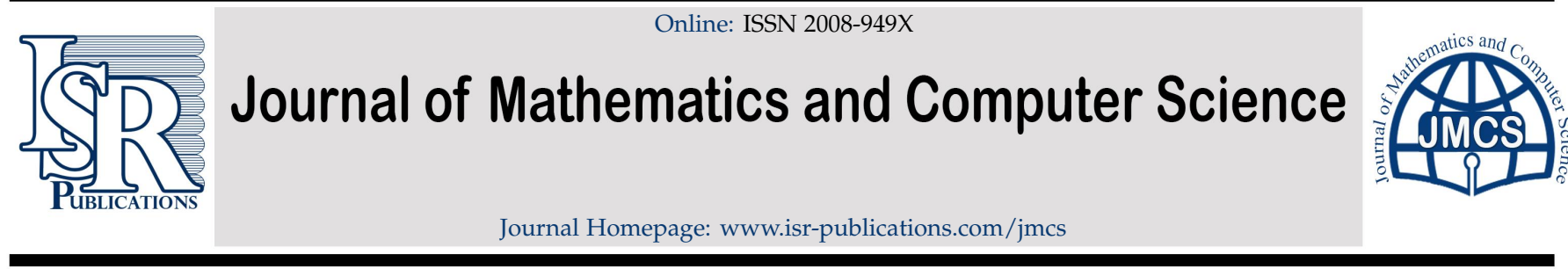

\title{
A general model of non-communicable diseases and its qual- itative analysis without finding the eigenvalues
}

\author{
Auni Aslah Mat Daud*, Toh Cher Qing \\ Faculty of Ocean Engineering Technology and Informatics, University Malaysia Terengganu, Kuala Nerus, 21030, Malaysia.
}

\begin{abstract}
Obtaining the analytical solutions of a linear ordinary differential equations is impossible without finding the eigenvalues. In this study, a general linear model of non-communicable disease (NCD) is formulated using the compartmental analysis and its qualitative properties are analyzed without finding the eigenvalues. NCDs are diseases which are not passed from person to person. The proof of the qualitative properties of the general model including the existence and uniqueness of its solution and equilibrium, and the positivity and boundedness of its solutions are provided. The global stability of the general model is analyzed using the theorem of compartmental matrix and Lyapunov function. It is found that the model has one unique non-negative equilibrium which is globally exponentially stable. As a real-world example, the general model and its qualitative analysis are implemented to a NCD, namely venous thromboembolism (VTE) among pregnant and postpartum women. VTE is selected in this study as it is a major global health burden due to its association with disability and lower quality of life and death.
\end{abstract}

Keywords: Mathematical modelling, qualitative analysis, non-communicable disease, venous thromboembolism, global stability, compartmental analysis.

2020 MSC: 97M10, 92D25, 37N15.

(C)2022 All rights reserved.

\section{Introduction}

This study focuses on the qualitative analysis of the linear population models. We are concerned only with a system which is assumed to be adequately described by a system of constant coefficient linear differential equations, namely the population dynamics of non-communicable diseases (NCDs) such as diabetes mellitus and hypertension. NCDs are diseases which are not passed from person to person. NCDs are also known as chronic diseases, such as cardiovascular diseases (like heart attacks and stroke), cancers, chronic respiratory diseases (such as chronic obstructive pulmonary disease and asthma) and diabetes. They are currently leading causes of morbidity and mortality worldwide and major Public Health challenges which increase the burden of infective diseases, especially for developing or lowand-middle income countries. Unlike infectious diseases, mathematical modelling is seldom applied to NCDs. It has been shown recently that the population dynamics of NCDs can be described and are better modelled by linear systems [2, 11-14].

\footnotetext{
${ }^{*}$ Corresponding author

Email address: auni_aslah@yahoo.com (Auni Aslah Mat Daud)

doi: $10.22436 /$ jmcs.024.01.07

Received: 2020-04-16 Revised: 2020-09-16 Accepted: 2020-11-20
} 
A very recent study highlights several issues in the studies of NCDs, including the lack of NCDs models using differential equations, the wrongly formulated mathematical terms, and the ill-posedness of governing equations [12]. This study will attempt to address these issues. Three NCDs have been studied recently, in which the mathematical models of thyroid disorder [11], hypertension [13] and diabetes mellitus during pregnancy are proposed and studied using stability and sensitivity analysis [14]. However, to date, no mathematical model of VTE has been studied in the literature. Note that, while the discussion in this paper is that of NCDs, various other potential applications such as modeling the cycles of carbon and other biogeochemical elements, should be kept in mind.

One may argue that for linear ordinary differential equations (ODEs), the conventional methods, such as the Laplace transform method and variation of parameters, can be used to solve the ODEs analytically. However, finding exact solutions of linear system of ODEs with constant coefficients involve solving characteristic polynomial. There is no general formula for finding the roots of a polynomial that is degree 5 or higher, especially if we deal with symbolic polynomials. It will require the aid of computer algebra system and frequently the form of solution given by a computer algebra system is large, cumbersome and hard to study. Even simplification routines may not help much [19]. Moreover, there are difficulties if the order of the linear equation or system is high [3]. In this study, we provide the qualitative analysis of linear systems without finding the eigenvalues. It is important to note that the methods of solving system of linear differential equations analytically is well established and described in many textbooks. It is also generally accepted that numerical techniques for linear differential equations yields acceptable solutions. Therefore, the discussion on the qualitative analysis of the model in this study is not an attempt to reinvent the wheel, and proof from previous studies will quoted whenever necessary. Qualitatively analyzing ODEs, means determining what the graph of a solution of an ODE (also called solution curve) looks like and approximating certain qualitative properties of ODEs, without finding the analytical or numerical solutions of the ODEs. The properties of ODEs includes the existence and uniqueness of solutions, stability, boundedness, etc.

The objectives of the present study are as follows. Firstly, to formulate a general population model which can be used to describe the dynamics of all NCDs. Secondly, to discuss the qualitative properties of the model, namely the existence of unique of its solution, the positivity and boundedness, and its stability. Finally, as an example of a real-world problem, the model will be used to study the population dynamics of patients with venous thromboembolism (VTE).

The organization of this paper is as follows. Section 2 discusses the formulation of the general linear modeling. In Section 3, the proof of qualitative properties of the model, namely the existence and uniqueness of the solution, the positivity and boundedness of the solution, and its global stability are provided. In Section 4, the general model and its qualitative analysis are implemented to a non-communicable disease, namely venous thromboembolism (VTE) among pregnant and postpartum women. Finally, some recommendations for future work is provided in Section 5.

\section{Formulation of the general NCD model}

A general linear model for such a population is developed using compartmental modeling (note that this is not a new idea, see for example [1]). Consider a heterogeneous population in which the epidemiological parameters for an individual may depend on certain factors (in this study, the factor is the stage of the NCDs), but can be grouped into finite homogeneous subpopulations. That is, individuals in a subpopulation are indistinguishable from one another (by homogeneous we imply that all of the individuals of a subpopulation is at all times homogeneous; there is instantaneous mixing with individual that enters the subpopulation). Therefore, the parameters may vary from subpopulation to subpopulation, but are identical for all individuals in a particular subpopulation. We begin the formulation of the general model by construction a general flow diagram which illustrates the population dynamics of linear model. In the flow diagram, the subpopulations are represented by the boxes while the arrows depict the possible 
movement of individuals between different subpopulations in the population. A common assumption is that the movements out of one subpopulation into the next one is governed by constant rates [16].

Figure 1 shows a general flow diagram which illustrates the population dynamics of a continuous-time linear model with $n$ subpopulations. Note that $N(t)=x_{1}+x_{2}+\cdots+x_{n}$ and $\mu_{i}=\mu+z_{i}$ where,

$x_{i}(t)$ represent the number of individuals in the subpopulation $x_{i}$ at time $t$;

$a_{i, j}$ represent the per capita transmission rate of individuals from the subpopulation $x_{i}$ to subpopulation $x_{j}$, which are assumed to be constants $\left(a_{i, j}=0\right.$ if there is no movement from subpopulation $x_{i}$ to subpopulation $x_{j}$ );

$c_{i}$ represent the number of individuals which enters subpopulation $x_{i}$;

$\mu$ represent the per capita death rate of the population;

$z_{i}$ represent the per capita rate of individuals who leave subpopulation $x_{i}$ (due to death and other factors), for $i=1,2, \cdots, n, j=1,2, \cdots, n$, and $i \neq j$.

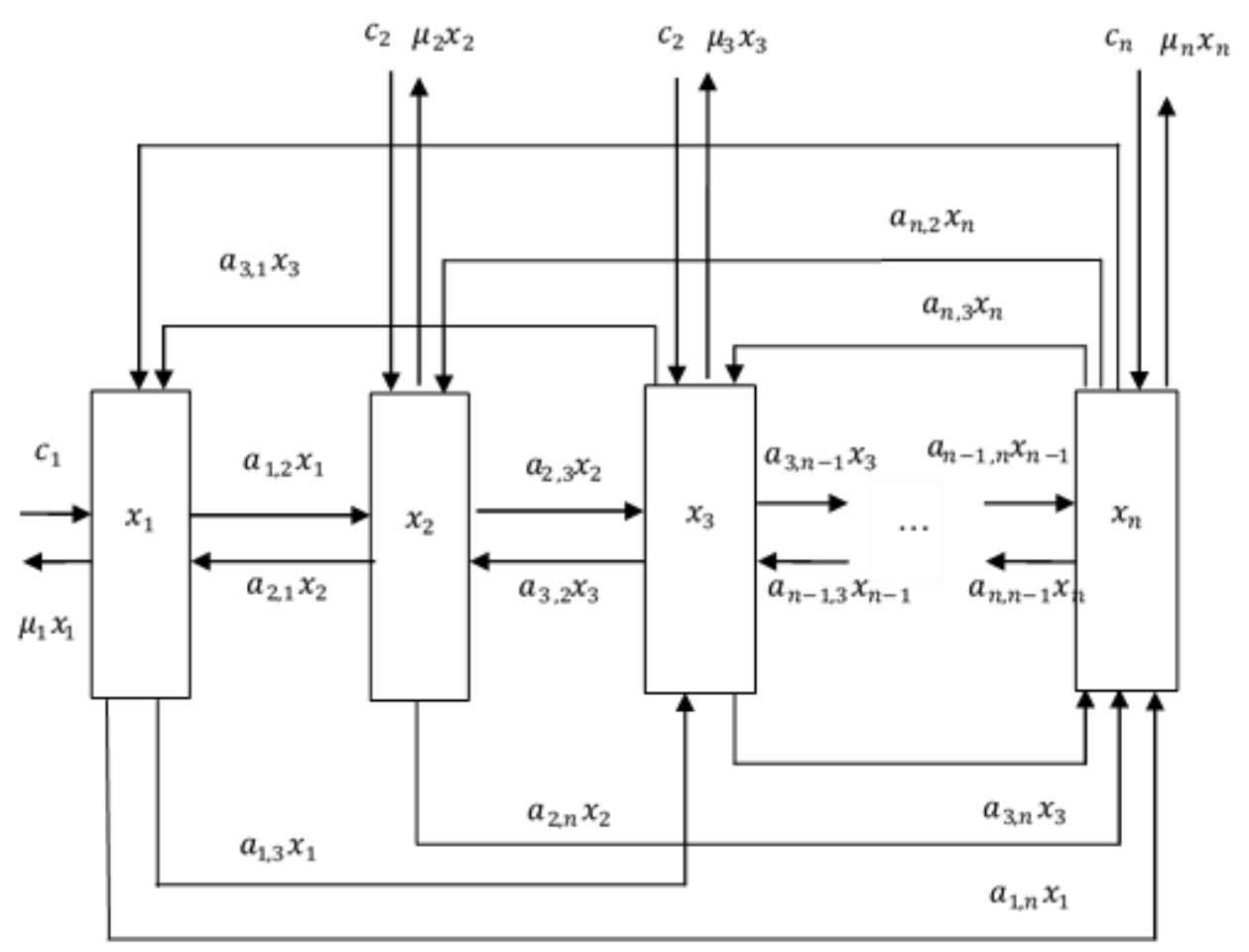

Figure 1: Flow diagram of the population dynamics of a NCD model with $n$ subpopulations.

Notice the underlying assumption that there are no time delays in the transfers: in the transfer from $j$ to $i$, as soon as individuals leave subpopulation $j$, it appears in subpopulation $i$. It is also assumed that the average time spent in subpopulation $i$ by individuals has a constant length $1 / \mu_{i}$. Based on Figure 1 , a general mathematical model can be formulated. The governing equations can be obtained by writing the instantaneous mass balance equation for each subpopulation. Using compartmental modelling [6], the general model of $n$ dimensional continuous-time system is governed by a linear system of $n$ differential equations

$$
\frac{d x_{i}}{d t}=c_{i}+\sum_{j=1}^{n} a_{j, i} x_{j}-\left[\sum_{j=1}^{n} a_{i, j}+\mu_{i}\right] x_{i},
$$

with initial conditions $x_{\mathfrak{i}}\left(t_{0}\right)=x_{\mathfrak{i}}(0) \in R$, for $i=1,2, \cdots, n$. 


\section{Qualitative properties of model (2.1)}

\subsection{Existence and uniqueness of solutions/equilbirum}

Model (2.1) can be written in matrix form $x_{i}^{\prime}=A x_{i}+c_{i}$, where

$$
A=\left[\begin{array}{ccccc}
-\tilde{a}_{1, j} & a_{2,1} & a_{3,1} & \cdots & a_{n, 1} \\
a_{1,2} & -\tilde{a}_{2, j} & a_{3,2} & \cdots & a_{n, 2} \\
a_{1,3} & a_{2,3} & -\tilde{a}_{3, j} & \cdots & a_{n, 3} \\
\vdots & \vdots & \vdots & \ddots & \vdots \\
a_{1, n} & a_{2, n} & a_{3, n} & \cdots & -\tilde{a}_{n, j}
\end{array}\right], \quad x_{i}=\left[\begin{array}{c}
x_{1} \\
x_{2} \\
x_{3} \\
\vdots \\
x_{n}
\end{array}\right], \quad c_{i}=\left[\begin{array}{c}
c_{1} \\
c_{2} \\
c_{3} \\
\vdots \\
c_{n}
\end{array}\right],
$$

and

$$
\tilde{a}_{k, j}=\sum_{i \neq j, j=1}^{n} a_{k, j}+\mu_{k}
$$

Based on the given definition of $a_{i, j}$ in Model (2.1), all elements of matrix $A$ are constant which represent the per capita transmission rates of individuals from subpopulation $x_{i}$ to subpopulation $x_{j}$. Therefore, the elements of matrix $A$ always exist and continuous hence the initial value problem $x_{i}^{\prime}=$ $A x_{i}+c_{i}, x\left(t_{0}\right)=x_{0}$ has a unique solution.

The existence of the equilibrium of Model (2.1), $\left(x_{1}^{*}, x_{2}^{*}, \cdots, x_{n}^{*}\right)$ depends on the nature of matrix $A$. From linear algebra, we know that Model (2.1) has exactly one equilibrium point if the determinant of matrix $A$ is non-zero, that is $|A| \neq 0$. From (3.1), we know that matrix $A$ is a diagonal dominant matrix, therefore matrix $A$ is nonsingular [8] and hence Model (2.1) has one unique equilibrium given by the constant vector $x_{i}^{*}=-A^{-1} c_{i}$.

\subsection{Positivity and boundedness of the solutions}

Since all the dependent variables in the general models denote physical quantities (in population models, they represent the number of individuals of a subpopulation), the negative solutions are not biologically meaningful. Therefore, we require that solutions that start from nonnegative initial conditions remain nonnegative for all time. Furthermore, if a model is to make a plausible prediction, a population should not be able to continue growing indefinitely (i.e. bounded).

Theorem 3.1. Positivity of the solutions of Model (2.1).

Proof. Since $c_{i}$ and $x_{i}$ represent the number of individuals, then $c_{i}$ and initial conditions $x_{i}\left(t_{0}\right)=x_{1}(0)$, $c_{i} \geqslant 0, x_{i}(0) \geqslant 0, i=1,2, \cdots, n$ are always non-negative, i.e., $c_{i} \geqslant 0$ and $x_{i}(t) \geqslant 0$, $(t) \geqslant 0$ for $i=1,2, \cdots, n$.

From (2.1), we know

$$
\frac{d x_{i}}{d t}+\left[\sum_{j=1}^{n} a_{i, j}+\mu_{i}\right] x_{i}=c_{i}+\sum_{j=1}^{n} a_{i, j} x_{j}, \quad i, j=1,2, \cdots, n .
$$

Hence,

$$
\frac{d x_{i}}{d t}+\left[\sum_{j=1}^{n} a_{i, j}+\mu_{i}\right] x_{i} \geqslant 0 .
$$

As inequality (3.2) is linear and first order, it can be solved using integrating factor method to yield

$$
\frac{d}{d t}\left[x_{i} e^{k_{1} t}\right] \geqslant 0 ; k_{1}=\left[\sum_{j=1}^{n} a_{i, j}+\mu_{i}\right] \text {. }
$$


Integrating inequality (3.3) with respect to $t$, and solving, we obtain

$$
x_{i}(t) \geqslant x_{i}(0) e^{-k, t}>0
$$

Therefore $x_{i}(t) \geqslant 0$ for $(t) \geqslant 0$. i.e., the solutions of Model (2.1) are always non-negative.

Alternatively, the general solution of initial value problems for the non-homogeneous constant coefficient linear system is given by variation of constants formula [14]

$$
x_{i}=e^{A t} x_{i}(0)+e^{A t} \int_{0}^{t} e^{-A s} c_{i} d s .
$$

It is not hard to see that solutions of this system that start positive stay positive in the future. Indeed (3.4) implies that if $x(0) \geqslant 0$, then $x(t) \geqslant 0, t \geqslant 0, e^{A t} \geqslant 0, t \geqslant 0$, and $e^{A(t-s)} \geqslant 0,0 \leqslant s \leqslant t$.

Theorem 3.2 (This is the exact statement of [1, Corollary 2.11]). Boundedness of solutions of Model (2.1).

Proof. To prove the boundedness of all $x_{i}(t)$, it is suffice to show that $N$ is bounded.

From (2.1),

$$
\begin{aligned}
\frac{d N}{d t} & =\frac{d x_{1}}{d t}+\frac{d x_{2}}{d t}+\cdots+\frac{d x_{n}}{d t} \\
& =\sum_{i=1}^{n} c_{i}+\sum_{i=1}^{n} \sum_{j=1}^{n} x_{i, j} x_{i}-\sum_{i=1}^{n} \sum_{j=1}^{n} x_{j, i} x_{j}-\sum_{i=1}^{n} d_{i} x_{i} \\
& =\sum_{i=1}^{n} c_{i}+\sum_{i=1}^{n} \sum_{j=1}^{n} a_{i, j} x_{i}-\sum_{i=1}^{n} \sum_{j=1}^{n} a_{j, i} x_{j}-\sum_{i=1}^{n}\left(\mu+z_{i}\right) x_{i} .
\end{aligned}
$$

Since $c_{i}$ and $z_{i}$ are always non-negative, and $a_{i, j} x_{i}=a_{j, i} x_{j}$, we get

$$
\frac{d N}{d t}+n \mu N \leqslant k_{2}, \quad k_{2}=\sum_{i=1}^{n} c_{i} .
$$

As inequality (3.5) is linear and first order, it can be solved using integrating factor method to yield

$$
\frac{\mathrm{d}}{\mathrm{dt}}\left[\mathrm{N} e^{\mathrm{n} \mu \mathrm{t}}\right] \leqslant \mathrm{k}_{2} e^{\mathrm{n} \mu \mathrm{t}}
$$

Integrating inequality (3.6) with respect $t$,

$$
N(t) \leqslant \frac{k_{2}}{n \mu}\left(1-e^{-n \mu t}\right)+N(0) e^{-n \mu t} .
$$

Taking limit when $t \rightarrow \infty$, we obtain

$$
N(t) \leqslant \frac{k_{2}}{n \mu} .
$$

Therefore, the total population $\mathrm{N}=\mathrm{x}_{1}+\mathrm{x}_{2}+\cdots+\mathrm{x}_{\mathrm{n}}$ is bounded since the number of individuals in every subpopulation $x_{i}, i=1,2, \cdots, n$ are bounded.

It is crucial to note that Model (2.1) will be analyzed in a feasible region D given by

$$
\mathrm{D}=\left\{\left(x_{1}, x_{2}, \cdots, x_{n}\right) \in R_{+}^{n}: 0 \leqslant N=x_{1}+x_{2}+\cdots+x_{n} \leqslant \frac{k_{2}}{n \mu}\right\},
$$

which can be verified to be positively invariant with respect to Model (2.1). In what follows, the model is epidemiologically and mathematically well-posed in D [7]. 


\subsection{Global stability analysis of Model (2.1)}

Without further transformations (which require the determination of eigenvalues of the coefficient Matrix A), the representation of the solution of (3.4) does not provide clear insight into how the solution behave for high dimension system, particularly in symbolic polynomials. The following theorem will provide the information about the global stability of Model (2.1), using two proofs, both without explicitly finding the eigenvalues of $A$.

Theorem 3.3. The NCD linear Model (2.1) is globally exponentially stable in D.

Proof 1: Using compartmental matrix. The real proof of this statement is based on Gershgorin's Circle Theorem, the proof of which relies on the strict negativity of the eigenvalues of the matrix. Therefore, the proof using compartmental matrix is not reproduced here. For linear systems, this implies globally asymptotically stable and hence globally exponentially stable.

Proof 2: Using Lyapunov Direct Method. (Note that it is well known (see for example [5, Theorem 2.10]) that in linear systems the strict negativity of all eigenvalues and the system's stability are equivalent. Originally this was proved by Lyapunov himself in 1892.)

Consider quadratic Lyapunov function

$$
V(x)=\frac{1}{2}\left(x_{1}^{2}+x_{2}^{2}+\cdots+x_{n}^{2}\right)=\frac{1}{2} \sum_{i=1}^{n} x_{i}^{2} .
$$

It is easy to see that $V(x)>0$ for $x \neq 0$ and $V(0)=0$. Furthermore, the derivative is

$$
\frac{d V}{d t}=\sum_{i=1}^{n} x_{i} \dot{x}_{i}=\sum_{i=1}^{n} x_{i}\left[c_{i}+\sum_{j=1}^{n} a_{j, i} x_{j}-\left[\sum_{i=1}^{n} a_{i, j} x_{i}+\mu_{i}\right] x_{i}\right]<0,
$$

for $x \neq 0$, since $a_{i, j} x_{i}=a_{j, i} x_{j}, N \leqslant \frac{k_{2}}{n \mu}$ (from Theorem 3.2) and there is at least one $z_{i} \neq 0$ (that is, NCDs can cause death). Besides, $\dot{V}(0)=0$. Therefore, Model (2.1) is globally asymptotically stable and hence globally exponentially stable.

\section{Application to VTE disorder among pregnant and postpartum women}

As an example, the findings of the previous sections will be implemented to a non-communicable disease, namely VTE among pregnant model. VTE is a condition where blood clots form in the vein, which is called deep vein thrombosis (DVT). The blood clot may dislodge, travel to the lungs and block the lung's blood vessel, known as pulmonary embolism (PE) that may be fatal. Risk factors for VTE are grouped into three main factors: changes in blood composition, disruption in blood flow and injury to the vessel wall [15]. Pregnant and post-partum women have an increased risk of blood clots forming due to the physiological increase in blood clotting factors composition (this is called hypercoagulable state) combined with dilation of vein, mechanical obstruction of blood flow by the enlarged uterus, vessel injury during childbirth and immobility. The presence of non-pregnancy related risks such as thrombophilia, lupus, sickle cell, heart disease, and other associated conditions further increase the risk of VTE. The combination of these factors increases the likelihood of VTE between four to five times during pregnancy and twenty times during postpartum period (up to eight weeks after termination of pregnancy) [4, 9]. VTE is a major global burden regardless of the socioeconomic status of the countries due to its association with disability, lower quality of life and death [18]. Among pregnant and post-partum women, VTE affects from 90 to 171 per 100,000 pregnancies [17, 18] with 714 deaths per 100,000 VTE cases [10]. VTE is not a leading cause of maternal deaths in developing countries as other causes predominate. However, in developed countries where those cases are successfully managed, VTE is a leading contributor to maternal deaths $[4,18]$. 


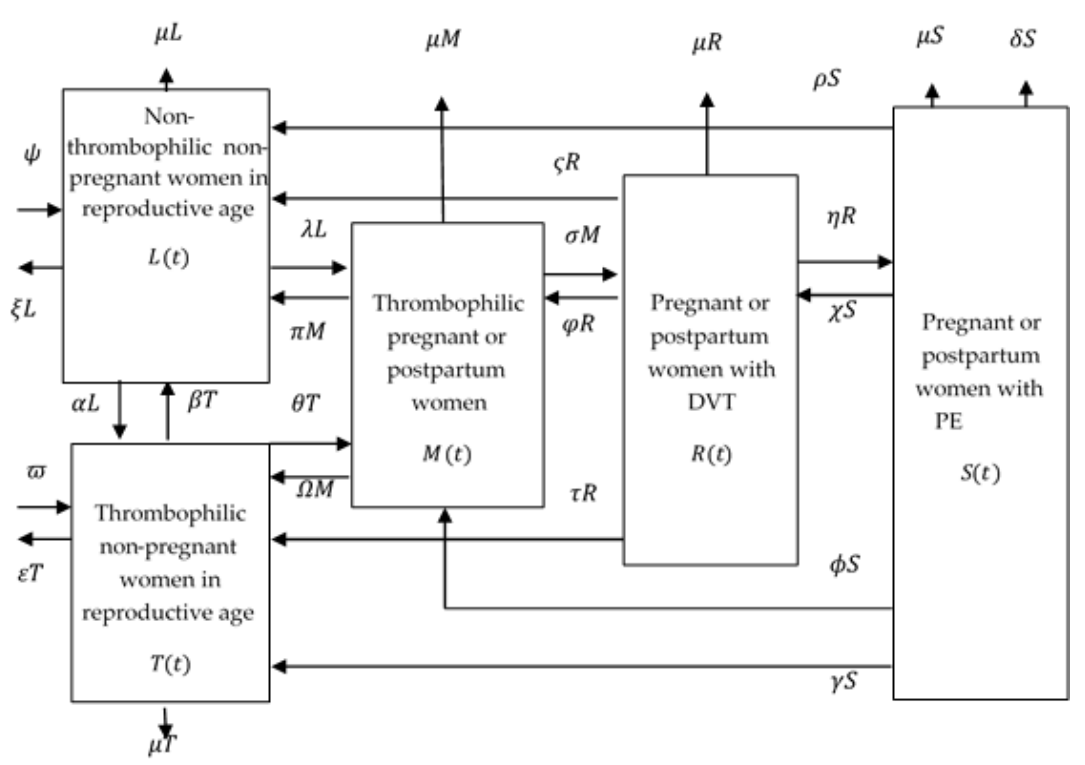

Figure 2: Flow diagram of the population dynamics of VTE during pregnancy.

Women can only become pregnant during the reproductive age. A woman in the hypercoagulable state (due to various health conditions stated above) will continue to be in that state during pregnancy and postpartum. However, there are normal women who only become hypercoagulable during pregnancy and postpartum due to pregnancy-related and postpartum-related conditions. Among these women, there are two possible implications after the postpartum period; (1) hypercoagulable state ceases as the risks factors are removed, or (2) hypercoagulable state continues if the risk factors persist (as an example, the dilation of vein started during pregnancy and then venous insufficiency developed and continued after postpartum). Venous thrombosis can occur in women in hypercoagulable state. When venous thrombosis occurs, it may (1) resolve, or (2) lead to PE which may be fatal. When a thrombus or an embolus resolves, depending on the presence of risk factors, the woman can be in hypercoagulable state (if the risk factors persist) or normal state (if the risk factors have been removed).

The flow diagram of VTE during pregnancy is shown in Figure 2. Five dependent variables represent subpopulations of different stages of the disease, namely $L(t)$ is the number of normal non-pregnant women in reproductive age, $M(t)$ is the number of thrombophilic pregnant or postpartum women, $R(t)$ is the number of pregnant or postpartum women with DVT, $S(t)$ is the number of pregnant or postpartum women have PE and $T(t)$ is the number of normal non-pregnant women in reproductive age population with thrombophilia. Using the notations in Section 3.1, $x_{1}=L(t), x_{2}=M(t), x_{3}=R(t), x_{4}=S(t)$, $x_{5}=T(t)$. The parameters in the models are described in Table 1 (all other $c_{i}$ and $a_{i, j}$ are zeros).

Therefore, the mathematical model of VTE during pregnancy is as follows:

$$
\begin{aligned}
\frac{d \mathrm{~L}}{\mathrm{dt}} & =\psi-(\xi+\mu+\kappa+\lambda) \mathrm{L}+\beta \mathrm{T}+\pi \mathrm{M}+\sigma \mathrm{R}+\rho \mathrm{S}, \\
\frac{\mathrm{dT}}{\mathrm{dt}} & =\varpi-(\varepsilon+\mu+\beta+\theta) \mathrm{T}+\alpha \mathrm{L}+\Omega \mathrm{M}+\tau \mathrm{R}+\gamma \mathrm{S}, \\
\frac{\mathrm{d} M}{\mathrm{dt}} & =\lambda \mathrm{L}-(\pi+\Omega+\mu+\sigma) \mathrm{M}+\theta \mathrm{T}+\varphi \mathrm{R}+\phi \mathrm{S}, \\
\frac{\mathrm{dR}}{\mathrm{dt}} & =\sigma \mathrm{M}-(\varphi+\tau+\mu+\eta+\sigma) \mathrm{R}+\chi \mathrm{S}, \\
\frac{\mathrm{d} S}{\mathrm{dt}} & =\eta \mathrm{R}-(\chi+\mu+\delta+\phi+\gamma+\rho) \mathrm{S},
\end{aligned}
$$

with initial conditions $L(0)=L_{0}, T(0)=T_{0}, M(0)=M_{0}, R(0)=R_{0}, S(0)=S_{0}$. 
Using the results in previous sections, we know that the VTE model has a unique non-negative equilibrium solution, given by the constant vector $x_{i}^{*}=-\hat{A}^{-1} c_{i}, i=1,2, \cdots, 5$, which is globally exponentially stable, where $\hat{A}$ is the coefficient matrix of the model.

Table 1: The parameters in the VTE model.

\begin{tabular}{|c|c|}
\hline Parameters & Description \\
\hline$\mu_{2}=\mu_{3}=\mu$ & death rate not due to VTE \\
\hline$z_{4}=\delta$ & death rate of pregnant/postpartum women due to PE \\
\hline$c_{1}=\psi$ & rate of women entering reproductive age without thrombophilia \\
\hline$z_{1}=\xi$ & $\begin{array}{l}\text { rate of women leaving the normal non-pregnant women in the reproduc- } \\
\text { tive age population without thrombophilia }\end{array}$ \\
\hline$c_{5}=\varpi$ & rate of women entering reproductive age with thrombophilia \\
\hline$z_{5}=\varepsilon$ & $\begin{array}{l}\text { rate of women leaving the normal non-pregnant women in reproductive } \\
\text { age population with thrombophilia }\end{array}$ \\
\hline $\mathrm{a}_{1,5}=\mathrm{k}$ & rate of normal non-pregnant women becoming thrombophilic \\
\hline$a_{5,1}=\beta$ & rate of thrombophilic non-pregnant women recovering from thrombophilia \\
\hline$a_{1,2}=\lambda$ & $\begin{array}{l}\text { rate of normal non-pregnant women becoming thrombophilic pregnant or } \\
\text { postpartum women }\end{array}$ \\
\hline$a_{5,2}=\theta$ & $\begin{array}{l}\text { rate of thrombophilic non-pregnant women becoming thrombophilic preg- } \\
\text { nant or postpartum women }\end{array}$ \\
\hline $\mathrm{a}_{2,1}=\pi$ & $\begin{array}{l}\text { rate of thrombophilic pregnant or postpartum women recovering from } \\
\text { thrombophilia become normal non-pregnant women }\end{array}$ \\
\hline $\mathrm{a}_{2,5}=\Omega$ & $\begin{array}{l}\text { rate of thrombophilic pregnant or postpartum women becoming throm- } \\
\text { bophilic non-pregnant women after postpartum }\end{array}$ \\
\hline$a_{3,1}=\sigma$ & $\begin{array}{l}\text { rate of pregnant or postpartum women with DVT recovering from DVT } \\
\text { and becoming normal non-pregnant women }\end{array}$ \\
\hline$a_{3,5}=\tau$ & $\begin{array}{l}\text { rate of pregnant or postpartum women with DVT recovering from DVT } \\
\text { but continue to be thrombophilic after postpartum }\end{array}$ \\
\hline$a_{2,3}=\sigma$ & rate of thrombophilic pregnant or postpartum women developing DVT \\
\hline$a_{3,2}=\varphi$ & $\begin{array}{l}\text { rate of pregnant or postpartum women with DVT recovering from DVT } \\
\text { but continue to be thrombophilic pregnant or postpartum women }\end{array}$ \\
\hline$a_{3,4}=\eta$ & rate of pregnant or postpartum women with DVT developing PE \\
\hline$a_{4,2}=\phi$ & $\begin{array}{l}\text { rate of pregnant or postpartum women with PE recovering from PE but } \\
\text { continue to be thrombophilic pregnant or postpartum women }\end{array}$ \\
\hline$a_{3,4}=x$ & $\begin{array}{l}\text { rate of pregnant or postpartum women with PE recovering from PE but } \\
\text { still not recovering from DVT }\end{array}$ \\
\hline$a_{4,5}=\gamma$ & $\begin{array}{l}\text { rate of pregnant or postpartum women with PE recovering from PE be- } \\
\text { coming thrombophilic non-pregnant women after postpartum }\end{array}$ \\
\hline$a_{4,1}=\rho$ & $\begin{array}{l}\text { rate of pregnant or postpartum women with PE recovering from PE be- } \\
\text { coming normal non-pregnant women }\end{array}$ \\
\hline
\end{tabular}

\section{Conclusions}

Obtaining the analytical solutions of a linear ordinary differential equations is impossible without finding the eigenvalues. The present study proposes a general linear model which describes the population dynamics of NCDs (and several other potential applications) using the compartmental analysis. The model has a unique non-negative equilibrium which is globally exponentially stable. This means that all solutions of Model (2.1) will converge exponentially to the equilibrium given by $x_{i}^{*}=-A^{-1} c_{i}$, 
independently of the initial conditions. Furthermore, the qualitative properties of Model (2.1) including the positivity and boundedness of the solutions are studied. The global behavior of the model is studied and analyzed using the theorem of compartmental matrix and Lyapunov function, without explicitly determining the eigenvalues of the coefficient matrix. Finally, the proposed model is applied to a realworld example, namely the VTE among pregnant and postpartum women. For further study, we intend to considerably extend this study by developing corresponding model with time delay to better describe the nature of the dynamics of NCDs.

The novel contributions of this study are the formulation of the general NCD model, the qualitative analysis on the properties (the existence, uniqueness, positivity and boundedness) of the solutions of the model and the study of VTE using the developed model and its analysis. The implementation of the formulated model and its analysis to VTE is due to its importance which has not been studied before. VTE is selected as it is a major global health burden due to its association with disability and lower quality of life and death.

\section{Acknowledgment}

This research is funded by Fundamental Research Grant Scheme (FRGS59523) of Ministry of Education, Malaysia.

\section{References}

[1] D. H. Anderson, Compartmental modeling and tracer kinetics, Springer-Verlag, Berlin, (1983). 2, 3.2

[2] A. Boutayeb, E. H. Twizell, K. Achouayb, A. Chetouani, A mathematical model for the burden of diabetes and its complications, Biomed. Eng. Online, 3 (2004), 8 pages. 1

[3] F. Brauer, J. A. Nohel, The qualitative theory of ordinary differential equations: An introduction, Dover Publications, New York, (1969). 1

[4] J. Drife, Thromboembolism: reducing maternal death and disability during pregnancy, Br. Med. Bull., 67 (2003), 177-190. 4

[5] K.-J. Engel, R. Nagel, One-Parameter Semigroups for Linear Evolution Equations, Springer-Verlag, New York, (2000). 3.3

[6] G. P. Garnett, An introduction to mathematical models in sexually transmitted disease epidemiology, Sex. Transm. Infect., 78 (2002), 7-12. 2

[7] H. W. Hethcote, The mathematics of infectious diseases, SIAM Rev., 42 (2000), 599-653. 3.2

[8] J. A. Jacquez, C. P. Simon, Qualitative theory of compartmental systems, SIAM Rev., 35 (1993), 43-79. 3.1

[9] A. H. James, Venous thromboembolism in pregnancy, Arterioscler. Thromb. Vasc. Biol., 29 (2009), 326-331. 4

[10] S. Liu, J. Rouleau, K. S. Joseph, R. Sauve, R. M. Liston, D. Young, M. S. Kramer, Epidemiology of pregnancy-associated venous thromboembolism: a population-based study in Canada, J. Obstet. Gynaecol. Can., 31 (2009), 611-620. 4

[11] A. A. Mat Daud, Mathematical modeling and stability analysis of population dynamics, In Dynamical Systems, Bifurcation Analysis and Applications, Springer, Singapore, 295 (2018), 3-13. 1

[12] A. A. Mat Daud, Some issues on the mathematical modeling of population dynamics using differential equations, Int. J. Math. Comput. Sci., 15 (2020), 501-513. 1

[13] A. A. Mat Daud, C. Q. Toh, S. Saidun, A mathematical model to study the population dynamics of hypertensive disorders during pregnancy, J. Interdiscip. Math., 22 (2019), 433-450. 1

[14] A. A. Mat Daud, C. Q. Toh, S. Saidun, Development and analysis of a mathematical model for the population dynamics of Diabetes Mellitus during pregnancy, Math. Models Comput. Simul., 12 (2020), 620-630. 1, 3.2

[15] P. H. Reitsma, H. H. Versteeg, S. Middeldorp, Mechanistic view of risk factors for venous thromboembolism, Arterioscler. Thromb. Vasc. Biol., 32 (2012), 563-568. 4

[16] C. I. Siettos, L. Russo, Mathematical modeling of infectious disease dynamics, Virulence, 4 (2013), 295-306. 2

[17] A. A. Sultan, J. West, L. J. Tata, K. M. Fleming, C. Nelson-Piercy, M. J. Grainge, Risk of first venous thromboembolism in and around pregnancy: A population-based cohort study, Br. J. Haematol., 156 (2012), 366-373. 4

[18] R. A. Virkus, E. C. L. Løkkegaard, T. Bergholt, U. Mogensen, J. Langhoff-Roos, Ø. Lidegaard, Venous thromboembolism in pregnant and puerperal women in Denmark 1995-2005: A national cohort study, Thromb. Haemost., 106 (2011), 304-309. 4

[19] S. A. Wirkus, R. J. Swift, A course in ordinary differential equations (2nd ed.), CRC Press, New York, (2004). 1 